\title{
Avanzando hacia la protección integral de los derechos de la infancia: Una mirada al proyecto de ley que regula entrevistas grabadas en video y otras medidas de resguardo a menores de edad víctimas de delitos sexuales
}

\section{Advancing towards an integral protection of children's rights: A look at the bill that regulates videotaped interviews and other measures to safeguard child victims of sexual crimes}

\author{
Alejandra Ijurra, Ariana Soto, Catherine LaBrenz y Paulina Sepúlveda
}

\begin{abstract}
Resumen
La presente investigación tuvo por objetivo realizar un análisis comprensivo del proyecto de ley de Entrevistas Videograbadas en Chile, en relación a los procesos investigativos de Suecia e Israel, pues estos últimos cuentan con políticas de protección de la infancia e intervenciones basadas en evidencia, permitiendo contrastarlo con la realidad jurídica nacional.

El enfoque metodológico fue cualitativo, utilizando el método de estudio de casos, donde se aplicaron entrevistas semiestructuradas a expertos en la materia en cada uno de los paises en cuestión, siendo analizadas posteriormente en su contenido.

La comprensión y análisis de estos diversos contextos y experiencias nos permite sugerir, a la luz del proyecto de ley de Entrevistas Videograbadas propuesto para Chile, que se requiere, en primer lugar, responder al compromiso adquirido al adscribir a la Convención Internacional de los Derechos del Niño con la creación de una política de protección integral para el resguardo de estos. Esta tarea implica cambios paradigmáticos importantes, que deben ir de la mano con la definición exacta de roles de las distintas instituciones que se involucran tras realizar denuncias por abuso sexual infantil. Y a su vez, aprender de la experiencia internacional, que nos indica que es preciso investigar y contextualizar las intervenciones desde prácticas basadas en evidencia, tomando a cada niño en su particularidad.
\end{abstract}

\footnotetext{
Abstract

This study aims to perform a comprehensive analysis of the bill of videotaped interviews in Chile by comparing it to the investigative processes implemented in Sweden and Israel. Since the latter countries have child protection policies and evidence-based intervention approach, they are thought to will serve as a contrast to compare the national legal reality. Using a qualitative approach, and a case study method, content analysis of semi-structured interviews with field experts in each of this countries was conducted. In light of the results we suggest that Chile should first commit to the International Convention on Children's Rights by creating a comprehensive protection policy to protect them. This task involves an important paradigmatic shift, which should also be supported by a clear definition of institutional roles in cases of alleged Child sexual abuse. International experience also suggests that the investigation process should be grounded on an evidence-based practice approach, taking into account children's particular traits.
} 


\section{Introducción}

La reflexión que impulsó este estudio tiene como base la relevancia de evitar la revictimización de niñas y niños en los procesos de investigación para casos de abuso sexual, que en muchas ocasiones generan un impacto de mayor permanencia que la propia vivencia abusiva que les llevó a interponer la denuncia.

Bajo esta lógica, recopilar información de otros países que han implementado la técnica de entrevista única o videograbada en el proceso de investigación, como son Suecia e Israel, permite a los profesionales que se insertan en este tipo de problemáticas comprender no sólo cómo se articulan los procesos legales, sino también considerar que estos llevan a la mano una serie de cambios socioculturales del pensar y actuar en directa relación con los derechos de las niñas y niños.

Quienes intervienen en casos de abuso sexual infantil en sus distintos procesos legales y reparatorios se ven enfrentados al dilema de balancear el interés superior del niño -evitando la victimización secundaria durante el proceso jurídico- con la necesidad de obtener el relato de éste dentro del juzgado para poder corroborar los hechos, respetando su derecho de ser escuchados.

En lo que a protección de derechos de la infancia respecta, Chile presenta una gran deuda políticojurídica. A la fecha, esto ha sido acallado por las instancias tomadoras de decisiones, realizando modificaciones parciales a la Ley 16.618, conocida como "Ley de Menores", que se encuentra vigente desde el año 1967. Mientras que, por otro lado, se manifiesta el imperativo social, por medio de distintas organizaciones sin fines de lucro, hacia la constitución de una Ley de Protección Integral de la infancia, el cual se constituye como un paso obligado que el país debe dar en su proceso de desarrollo no solo en infancia, sino también en materia de Derechos Humanos, respondiendo a los compromisos que ha adquirido en ello.

En Chile, tras 24 años de haber adscrito a la Convención de los Derechos del Niño (CDN), la infancia legislativamente sigue siendo mirada bajo la lupa de los "menores" y, por tanto, carentes, vulnerables y necesitados del tutelaje estatal.

Como consecuencia, los procesos de investigación en casos de abuso son altamente vulneradores para niñas y niños, viéndose expuestos a brindar un relato coherente de lo vivido para ser escuchados por la sociedad adultocéntrica, personificada en policías, fiscales, jueces y otros agentes presentes en el pro- ceso investigativo del delito. La prestación reiterada de declaraciones hacia diversos servicios motivó el proyecto de ley de Entrevistas Videograbadas del cual trata este escrito.

En este documento encontrará además las experiencias de Suecia e Israel en estos procedimientos, que han implicado avances políticos como también paradigmáticos donde la sociedad en sí vivió cambios estructurales para mirar al niño como sujeto de derechos y actuar de modo consecuente con ello.

\section{Marco de referencia \\ Consecuencias del Abuso Sexual Infantil}

El abuso sexual infantil es un fenómeno de larga data histórica. Desde tiempos remotos se utilizaba a la población infanto-juvenil para satisfacer fantasías sexuales del mundo adulto, cosificando la existencia de los mismos e invisibilizando sus necesidades. Ejemplo de ello son los denominados efebos que en la antigua Grecia correspondían a adolescentes hombres que por sus atributos físicos llevaban un mensaje erótico implícito (Alzate, 2002).

Si bien en la actualidad la tolerancia de la sociedad frente a estas situaciones es mucho menor que en el pasado, aún persisten estas conductas abusivas.

En este sentido, entenderemos por Abuso Sexual Infantil (en adelante ASI) a:
"[...] Contactos e interacciones entre un niño y un adulto cuando el adulto (agresor) usa al niño para estimularse sexualmente él mismo, al niño o a otra persona. El abuso sexual puede ser también cometido por una persona menor de 18 años cuándo ésta es significativamente mayor que el niño (víctima) o cuando el agresor está en una posición de poder o control sobre otro". (National Center of Child Abuse and Neglect, 1978; citado en Save the children, s/f) Dentro de esto también se considera el exhibicionismo.

Considerando la alta complejidad de las dinámicas abusivas, la afectación principal que dejan las conductas de este tipo en niñas, niños y adolescentes (en adelante NNA) es a nivel emocional: si bien la agresión irrumpe la intimidad de lo físico e indemnidad sexual, es la memoria traumática la que traduce esta vivencia en otras esferas del desarrollo, tal como se expresa en el siguiente extracto del proyecto de ley de Entrevistas Videograbadas para Chile:

Para un menor de edad, la agresión sexual se presenta con una fuerza desestabilizadora inconmensurable, involucrando aspectos 
afectivos, conductuales y relacionales altamente confusos, que no sabe cómo juzgar y ante los cuales no tiene las herramientas para responder adecuadamente. En consecuencia -aunque dependiendo de la entidad de la agresión, la etapa evolutiva del menor de edad y las circunstancias particulares del mismo o de la agresión-, los delitos sexuales suelen dejar una profunda y dolorosa huella en el desarrollo emocional, conductual, físico, social y cognitivo de las personas; en muchos casos, de difícil reparación. (Senado, 2014a)

Según la Encuesta Nacional de Opinión Pública realizada por la Universidad Diego Portales en 2005, el abuso sexual a NNA ha sido considerado como el delito más grave que puede afectar a una persona (Maffioletti y Huerta, 2011); dicha gravedad se relaciona con las distintas áreas que impactan, tanto en la experiencia directa como posteriormente en los procesos de denuncia e intervención judicial para perseguir y sancionar estas acciones.

Este abuso puede presentar consecuencias sintomáticas a corto plazo y en el desarrollo psicológico a largo plazo, distinguiéndose dos fases (Barudy, 2000; en Ibaceta, 2007):

Un proceso de traumatización coexistente con el abuso:

- Efectos sintomáticos a corto plazo.

- Mecanismos defensivos (por ejemplo, disociación) que permiten la sobrevivencia aun cuando parezcan desadaptativos.

Un proceso de adaptación o resocialización secundaria con efectos específicos (carreras morales):

- Efectos sintomáticos a largo plazo.

- Confrontación de los mecanismos defensivos.

- Impacto sobre el desarrollo psicológico y la construcción de la personalidad.

La gravedad de las consecuencias dependerá de una serie de factores, tales como la periodicidad del abuso, la edad del ofendido, si es intra o extrafamiliar, severidad de lesiones, entre otros aspectos.

Lo que sí es posible de afirmar, dentro del marco de las probabilidades, es que las consecuencias pueden ser agravadas por la reacción de un sistema judicial que abre procesos una y otra vez para obtener información una vez realizada la denuncia.

\subsection{Procedimiento penal en Chile para casos de Abuso Sexual Infantil}

En la reforma constitucional realizada en Chile en el año 1997, se crea el Ministerio Público, organis- mo encargado de dirigir todas las investigaciones de hechos constitutivos de delitos, dentro de estos también los de índole sexual, debiendo ejercer acción penal pública.

Si bien esta reforma significó un avance en los procesos investigativos de los delitos y la resolución satisfactoria del conflicto penal, ha sido bajo un costo emocional y social para las víctimas, que ha repercutido en pasar por los procesos de investigación con diferentes servicios, produciendo altas cifras de victimización por parte del sistema (Ministerio Público, 2012).

Como se menciona en la Guía de Entrevista Investigativa (Ministerio Público, 2012), en el año 2008 la Fiscalía Nacional define una serie de lineamientos estratégicos, orientados a mejorar la tarea constitucional iniciada en el año 1997; es decir, la persecución penal de los hechos constitutivos de delitos y además la protección de la víctima. Es así que en el plan estratégico del año 2009-2015 se estableció que los delitos sexuales, por una parte, constituyen una tipología delictiva necesaria de abordar y mejorar la calidad de la persecución penal $y$, por otra, considera que el abordaje de los delitos sexuales deben ser específicos y especializados, dado que la ocurrencia de estos delitos lo son en virtud del alto grado de vulnerabilidad de la víctima, principalmente mujeres y NNA. Sin embargo, en esta necesidad de resguardar los derechos humanos es que se cuestiona legalmente el relato de la víctima a fin de probar la culpabilidad del hasta entonces supuesto victimario, facilitando su revictimización. A su vez, en muchos casos las medidas de protección aplicadas a los NNA víctimas de delitos sexuales contribuyen a agravar las consecuencias del delito, alejándose del resguardo y protección que se busca con ello.

En el año 2012, la Fiscalía especializada de delitos sexuales y violentos elabora la "Guía de entrevista investigativa con niños, niñas y adolescentes víctimas de delitos sexuales" (GEV), cuyo objetivo es incrementar y mejorar la calidad de las investigaciones por delitos sexuales y disminuir la victimización secundaria del afectado por el delito, reduciendo la cantidad de entrevistas a las cuales es sometido.

Si pusiéramos en orden el proceso de declaraciones y entrevistas a las cuales son sometidos los NNA en Chile, podríamos mencionar: Declaración al momento de develar en el Servicio Médico Legal, luego con el Encargado de Brisexme, declaración ante la Brigada de Delitos Sexuales de la Policía de Investigaciones. Luego, entrevista con profesional de la unidad de atención a víctimas y testigos. También 
podemos agregar las pruebas periciales de daño y credibilidad y, por último, en caso de que sea derivado a terapia de reparación, nuevamente será expuesto a relatar los hechos.

Esta realidad dista mucho de los procesos que viven otros países que adscribieron a la Convención en el mismo año que Chile, pero que iniciaron acciones políticas legislativas concretas para materializar el interés superior del niño con base en el respeto de sus derechos. Y en lo que a delitos sexuales respecta, existen casos de profundos avances para evitar la revictimización penal del ofendido:

- En el Reino Unido, existe un mecanismo para proteger a NNA víctimas de delitos graves desde 1991. El juez evalúa, con anterioridad al juicio, si el menor está o no en condiciones de declarar, no permitiendo su declaración en caso negativo. Además, la declaración del menor es grabada y luego exhibida (San Martín, 2014);

- En España, la Ley de Enjuiciamiento Criminal también contempla, en su artículo 707, un procedimiento en virtud del cual se establece la entrevista grabada como mecanismo para evitar la revictimización (Senado, 2014b);

- En Canadá en el año 1988, el Parlamento promulgó la declaración C-15 e impulsó la enmienda del código penal a fin de reformar las tipificaciones de delitos y normas procesales específicas en casos de abusos de niños que implicaban agresión sexual. Estas reformas comprendieron el uso de asistencias testimoniales, tales como declaraciones grabadas en videos, mamparas y circuitos cerrados por televisión (CCTV) en aquellos casos en los que el testigo o víctima demandante fuese menor de dieciocho años. Las reformas procesales se proponían facilitar la entrega de las pruebas por parte del niño y además aliviar la revictimización que experimentaría al tener que repetir una y otra vez sus experiencias (Bala, 1990).

En cuanto a la prevalencia, según Mossige et al. (2007) los estudios internacionales arrojaron que: $27 \%$ de mujeres menores de 18 años reportaron haber tenido "indecent exposure" en sus vidas, autoinformado $9,2 \%$ de mujeres y 3,7\% de hombres menores de 18 años encuestados que habrían tenido relaciones sexuales bajo coerción. Priebe $\&$ Svedin (2009) concluyen que 13,5\% de mujeres y $5,6 \%$ de hombres en cuarto medio informan haber sido violados con penetración en un mínimo de 1 oportunidad.

Jones (1991) encontró distintos tipos de daños asociados a la victimización secundaria de niños que han vivido experiencias de ASI, incluyendo sobreintervención profesional, entrevistas repetidas, exámenes físicos repetitivos, asistencia mandatoria en el tribunal, los cuales tenían un impacto negativo en el bienestar del NNA.

Con respecto a las tasas de revictimización, Ceci \& Bruck (1995) arrojaron que un niño tendrá que relatar un promedio de 12 veces lo ocurrido durante el proceso legal.

De este modo, la relevancia de tomar medidas en este tipo de fenómeno se encuentra en que no corresponden a hechos aislados; por el contrario, se estima que en el contexto occidental, 1 de cada 3 niñas y 1 de cada 7 niños sufren de abusos sexuales antes de cumplir los 18 años, abarcando a un 8,7\% del total de NNA, siendo la edad promedio de abuso entre los 8 años y medio (Montoya et al., 2004; en UNICEF-UDP, 2006).

Según los datos del Ministerio Público (2014, citado en Senado, 2014b), el año 2013 en Chile hubo un incremento de las denuncias por delitos sexuales cometidos contra niños y niñas menores de 14 años, produciéndose 4.945 denuncias por abuso sexual con contacto.

Entre los años 2000 y 2013 hubo más de 177.000 denuncias por estos delitos, según los datos del Ministerio Público (2014, citado en Senado 2014a). SENAME indica que el ingreso por estas causas alcanza a más de 30.000 niños y adolescentes (Hernández, 2010). Y, según una publicación del diario La Tercera, de 39.639 agresiones sexuales contra menores, entre el 2011 y el 2013, sólo el 12\% (4.900) resultaron en condena (Senado, 2014b).

Estos antecedentes dan cuenta de la presencia de los niños y sus familias en los procesos investigativos que, de acuerdo a los antecedentes mencionados, requieren de la intervención del Estado para la protección de estos como sujetos de derechos.

Dicha intervención del Estado, por medio de sus diferentes instituciones (SENAME, tribunales, servicios de salud, Fiscalía), se ve afectada por la alta demanda de la acción penal y las carencias de estos servicios, no solo a nivel de infraestructura, sino que también de recursos humanos, implicando constantes derivaciones que terminan desgastando a las familias involucradas y agravan las condiciones emocionales de NNA ofendidos por delitos sexuales.

Actualmente, en la institucionalidad de la Fiscalía, programas de infancia y ONG, existe la voluntad de minimizar la victimización secundaria en los procesos de investigación penal, que va de la mano con 
un desafío aún mayor: el de generar cambios de paradigma en la concepción de infancia y la valoración de la niñez.

\section{Revictimización ${ }^{1}$ de NNA en los procesos penales}

La victimización secundaria en la que incurren los procesos penales "consiste en el sufrimiento que experimenta la víctima en su paso por las diferentes instancias del sistema procesal penal y por las reacciones de su entorno social, lo cual ha sido reconocido como uno de los efectos más nocivos de la victimización por las Naciones Unidas" (1999, citado en Senado, 2014a. p. 2). Esto se manifiesta en la toma repetida de declaraciones, en el sometimiento a múltiples peritajes, en la duda sobre la veracidad del relato de NNA, en la falta de información, en la no especialización de algunos funcionarios y en las inadecuadas instalaciones en las que deben declarar, por mencionar algunos aspectos.

Latinoamérica tampoco queda exenta de las reflexiones y de los procesos de reforma judicial, para entregar mayor eficacia a los procesos judiciales en materia de delitos sexuales.

Todo lo anterior tiene el objetivo de reducir lo más posible el estrés y evitar la revictimización, pero además favorecer la obtención de pruebas válidas, que mejoren la calidad y faciliten la realización de justicia.

En cuanto a estrategias para evitar la victimización secundaria de niños/as ofendidos/as sexualmente, UNICEF (2005) creó un manual de mejores prácticas en donde sugieren un programa de capacitación especializado para profesionales que intervienen o acompañan a niños/as ofendidos/as sexualmente.

En este mismo documento, se sugiere que el organismo estatal a cargo de las investigaciones y el proceso judicial, debería asegurar de que el niño/a no tenga que confrontar directamente al supuesto agresor, indicando que se debe "evitar el contacto directo entre una víctima infantil o testigo y el acusado en cualquier etapa del proceso judicial" (UNICEF, 2005, p. 14). En el artículo XIII del documento de UNICEF, refieren que "el investigador deberá, dentro de lo posible, evitar la repetición de la entrevista durante el proceso judicial, con el fin de evitar la victimización secundaria del niño" (UNICEF, 2005, p. 15).
Themeli \& Panagiotaki (2014) encontraron en su investigación que uno de los factores principales en la revictimización en NNA era la falta de experiencia y especialización del profesional que realiza la entrevista forense.

En distintos estudios (Themeli \& Panagiotaki, 2014; Pipe et al., 2010) se ha encontrado que la revictimización era minimizada cuando los profesionales que realizaron la entrevista tenían formación, especialización y experiencia en entrevistas forenses en la materia. Asimismo, la asignación de un profesional para acompañar al joven durante el proceso investigativo también facilitaba el relato y la experiencia, siendo eso parte del protocolo en Suecia, Israel y algunos estados de los Estados Unidos.

Cada vez que un niño o una niña deben repetir lo que le sucedió a los distintos servicios representa una nueva agresión que le afecta emocionalmente. Esto provocaría una sensación de indefensión y vulnerabilidad, a la vez que los reiterados interrogatorios y cuestionamientos le impiden superar la experiencia traumática, obstaculizando su recuperación.

Underwager \& Wakefield (1990), a partir de una serie de entrevistas realizadas a personas que habían vivenciado este tipo de abuso en su infancia, concluyeron que $20 \%$ de las entrevistadas señalan como daño principal a la reacción de terceros dentro del proceso investigativo, tanto por parte de la policía como de sus propias familias.

Bastaría poner un ejemplo del estudio de Jaudes \& Martone (1992), el cual arrojó que 50\% de los NNA en su muestra tuvieron 3 o más entrevistas forenses enfocado en la experiencia abusiva, y $76 \%$ con un mínimo de dos entrevistas forenses, requiriendo que revivieran el trauma en múltiples instancias.

En la actualidad en nuestro país, como medida para evitar este fenómeno, solo se permite la toma de declaración anticipada de víctimas de delitos sexuales menores de edad, la que se encuentra establecida en el Código Procesal Penal, art. 191 bis (Ministerio de Justicia, 2000). Sin embargo, esto no es suficiente para proporcionar protección de derechos durante el proceso penal, ya que, tal como se mencionó en el apartado anterior, la toma de declaración sigue siendo reiterativa y perversa en sí misma.

En otros países (Israel, Estados Unidos y Suecia, entre otros), se crearon Centros de Promoción

1 La revictimización hace referencia directamente a un sujeto puesto en una condición no libre ni voluntaria sino dada por el ejercicio de otro poder, que ejerce fuerza o presión. Se trata de un alguien que ha sido víctima, pero el prefijo "re" nos dice de la característica de esa condición su repetición. Por lo tanto, la revictimización es una palabra derivada que hace referencia a la experiencia que victimiza a una persona en dos o más momentos de su vida (Comisión Intereclesial de Justicia y Paz, 2010). 
Infantil, especializados para acompañar al NNA durante todo el proceso investigativo, jurídico y de reparación, con el fin de evitar la cantidad de personas que interrogan al NNA, siendo el profesional designado el responsable para coordinar y transmitir cualquier información o relato del niño hacia los demás actores.

El proyecto de ley de Entrevistas Videograbadas para Chile se sustenta en evitar este tipo de victimización, que parece ser más una nueva victimización que una victimización secundaria. En efecto, los niños y niñas vuelven a ser objeto de cuestionamiento y de análisis e, incluso, de todo tipo de manipulaciones, en un sentido y en otro, pues mientras Fiscalía busca verificar lo acontecido, la defensa siempre buscará una manera de reducir la responsabilidad del imputado.

\section{Experiencia de Suecia e Israel en procesos pena- les en casos de Abuso Sexual Infantil}

Países como Suecia e Israel llevan varios años con un modelo del proceso investigativo similar al propuesto en el Proyecto de Ley para Chile.

En el contexto de Suecia, desde el siglo XIX ha existido legislación y actas definiendo al abuso sexual como ilegal. Más recientemente, en el año 2005, el Acto de Delitos Sexuales fue aprobado y con ello se define el marco jurídico de los que constituyen Abusos Sexuales, especificando ciertos delitos en contra de NNA, fijando la edad del consentimiento según el Acta de 2005, desde los 15 años de edad (Amnesty International, 2006).

En cuanto al procedimiento, existen centros especializados para realizar entrevistas en donde hay una denuncia e investigación de un posible abuso sexual. En estos centros, un policía especializado realiza la entrevista con el NNA dentro de una sala de espejo. El fiscal y un equipo profesional compuesto por un psicólogo y trabajador social especializado observan la entrevista desde el otro lado del espejo. Todas las entrevistas realizadas por el policía son grabadas, para que solo tenga que relatar la experiencia abusiva una vez; por tanto, no tiene que confrontar directamente al supuesto agresor en el tribunal, ya que se incorpora la entrevista grabada.

Está en manos del fiscal decidir si seguirá con el proceso jurídico, y los profesionales determinan el plan de intervención o reparación, así como también determinan si hay suficiente evidencia para seguir y llevar el caso al tribunal, en donde hay un juez profesional y tres jueces elegidos de la comunidad, quienes deciden el veredicto y la sentencia.
Lo descrito es guiado por la Ley de Códigos Penal (Swedish Penal Code, 1999), en donde, en conjunto con el Acto de Delitos Sexuales, hay definiciones y descripciones de los diversos delitos sexuales que pudieran ser cometidos en contra de personas menores de 18 años.

En el caso de Israel, su Código Penal (1977) especifica la edad de consentimiento desde los 14 años, con leyes especiales para quienes estén en el rango de 14 y 16 años. Hay largas condenas para personas que han cometido delitos sexuales en contra de menores de edad, aumentando las sentencias en situaciones donde el ofensor tiene una posición del poder frente al ofendido (Law Library of Congress, 2014).

Según las estadísticas de los Centros de Delitos Sexuales en Israel, casi un 50\% de llamadas recibidas por casos de abuso sexual eran realizadas por personas menores de 18 años de edad, representando un porcentaje significativo de casos de ASI (Association of Rape Crisis Centers in Israel, 2007). Aunque según la ley de Israel, los ofendidos de violación son solo mujeres, otros tipos de ASI pueden ocurrir en contra de ambos sexos.

\section{Propuesta de Entrevistas Videograbadas en Chi- le: Sustentos con base en la reflexión del sistema internacional}

El Ministerio del Interior y Seguridad Pública y la Fundación Amparo y Justicia convocaron a una mesa de trabajo durante los años 2011 y 2012, la que tuvo representantes del Ministerio Público, del Poder Judicial, del Poder Legislativo, de asesores del "Proyecto U-REDES Infancia y Justicia" de las Facultades de Ciencias Sociales y de Derecho de la Universidad de Chile, y de asesores externos, tanto nacionales como extranjeros. En esta mesa se utilizaron los resultados de la encuesta "Percepción de los procesos de investigación y judicialización en los casos de agresiones sexuales infantiles", encargada en 2008 por la Fundación Amparo y Justicia y el Ministerio Público al Centro de Medición de la Pontificia Universidad Católica de Chile (MIDE UC), como los seminarios realizados en relación con la temática a estudiar (Senado, 2014a).

A raíz de las discusiones de esta mesa y del sentir colectivo acerca de lo victimizante del proceso penal en Chile es que se impulsa el proyecto de ley "Regulación de entrevistas en video y otras medidas de resguardo a menores víctimas de delitos sexuales". Éste tiene por objetivo principal "mejorar la forma en que un menor de edad que ha sido víctima de un delito sexual se relaciona con el Sistema Proce- 
sal Penal, de manera de minimizar la victimización secundaria que puede afectarle y darle un más efectivo resguardo a sus derechos" (Senado, 2014a).

La idea principal de este proyecto de ley es que los niños y niñas no declaren en los juicios orales, sino que se les entreviste en dos oportunidades antes del juicio y que éstas sean grabadas en video, una de ellas ante el fiscal (en un plazo de 72 horas una vez realizada la denuncia, a menos que haya un impedimento grave para su realización) y, posteriormente, como prueba anticipada para el juicio, esta última conducida por el juez de garantía. Ambas entrevistas serían realizadas por profesionales idóneos y capacitados para ello; en el marco de lo ideal, se espera que sean ejecutadas por el mismo profesional (Campaña "No me pregunten más", 2014), tal como se muestra en figura 1.

Se consideran también las circunstancias personales, emocionales, cognitivas, físicas, idiomáticas, étnicas, culturales y de género, por lo que sería un procedimiento estandarizado, posible de adecuar a las características individuales de cada una de las NNA. A su vez, no podrá citarse al menor de edad a declarar en el juicio oral, a menos que éste sea mayor de 14 años, quienes podrán declarar voluntariamente en calidad de víctimas y sujetos a las normas generales (Senado, 2014a).

Se contempla que todo peritaje de credibilidad del relato que se solicitare posteriormente tendrá que ser realizado desde el registro audiovisual de la entrevista, con ello se tiene el respaldo de no tener que volver a requerir del relato del niño (Senado, 2014a).

En caso de ser necesario para el bienestar del NNA, el Fiscal podría autorizar que esté acompañado de un adulto responsable, un intérprete o traductor en el momento de la entrevista, como también tendrá la facultad de suspender la entrevista o postergarla, si fuese necesario, para resguardar su integridad, para lo cual debe dejar constancia. Sin perjuicio de lo anterior, a pesar de no realizarse la entrevista investigativa, de todas formas podrá realizarse la entrevista judicial, a cargo del juez de garantía (Senado, 2014a).

Para estas entrevistas se utilizará una infraestructura adecuada (salas Gessel o de espejo), que cuenten con los implementos necesarios de acuerdo a la etapa del desarrollo y necesidades del entrevistado, para posteriormente ser transcritas en su integridad y ser utilizadas en el proceso de investigación del delito. Este contenido será reservado, regulando quiénes pueden acceder y sancionando la vulneración de reserva (Senado, 2014a).

Las ventajas de la inmediatez de la primera entrevista busca minimizar fallos relacionados con la memoria que podrían afectar el relato, como también el que éste sea más espontáneo y evitar que se vea contaminado por presiones (con peligro de retractación) o por haberlo repetido ya muchas veces antes (Senado, 2014a). Otra ventaja de la inmediatez de la entrevista consiste en poder comenzar tempranamente con el proceso reparatorio.

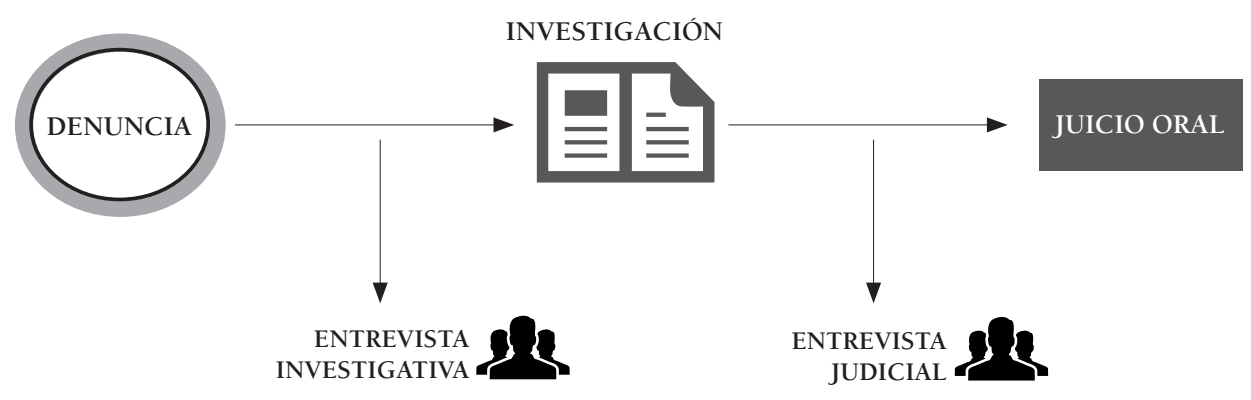


Sin embargo, Bruck, et al. (2007, p. 12) refiere: "la entrevista forense es un proceso de evaluación extendida de un niño, siempre cuando el niño tenga demasiado susto o sea demasiado joven para poder develar completamente sus experiencias en una entrevista forense preliminar". Asimismo, Hershkowitz \& Terner (2006) encontraron que al realizar una segunda entrevista forense, $25 \%$ de la información relatada era nueva y relevante a la develación y descripción del abuso, encontrando beneficioso la utilización de más de una entrevista forense.

Los hallazgos de las investigaciones realizadas en Suecia e Israel concluyeron que debería ser el mismo profesional quien realice todas las entrevistas grabadas de un niño o niña en particular, tratando dentro de lo posible de minimizar la cantidad de entrevistas realizadas (Bruck et al., 2007; Cross et al., 2007; Hershkowitz et al., 2006; Vandervort, 2006). En estos centros, los profesionales, sea un psicólogo o trabajador social, realiza la entrevista forense, utilizando técnicas basadas en evidencia para implementarla.

\section{Marco metodológico}

Esta revisión estuvo orientada por los siguientes objetivos:

\section{Objetivo general:}

- Realizar un análisis comprensivo del proyecto de ley de Entrevistas Videograbadas en Chile, en relación con los procesos investigativos de Suecia e Israel.

\section{Objetivos específicos:}

- Conocer los procesos de investigación en casos de abuso sexual infantil según la experiencia internacional.

- Identificar aspectos positivos y negativos de las entrevistas videograbadas a nivel internacional.

- Indicar sugerencias proyectivas al escenario que se propone generar el proyecto de ley de Entrevistas Videograbadas.

\section{Paradigma, enfoque, marco muestral y técnicas de recolección de datos}

La presente investigación se ajustó al paradigma interpretativo, ya que concibió la realidad como construida por los mismos individuos que la componen y por esto la comprensión de los significados y con ello los datos a conocer vienen dados por los mismos (Goetz \& LeCompte, 1988).
El enfoque fue cualitativo, dado que se apuntó al análisis de un escenario de la realidad donde interactúan e influyen distintos actores, no posibles de abarcar desde la perspectiva cuantitativa. Considerando principalmente los discursos como eje de la interpretación de los datos (Stake, 1998), que para el caso corresponden a los relatos de los profesionales de cada país.

Se utilizó un nivel descriptivo, pretendiendo reconocer las características de lo observado en situación natural, no experimental, por lo que las variables no fueron manipuladas (Goetz \& LeCompte, 1988), es decir, el análisis corresponderá solo a los países en cuestión, sin ansias de generalizar a la realidad de otros; de ahí que su naturaleza sea empírica.

Por su parte, el método fue el estudio de casos, ya que obtuvieron datos de Chile, Suecia e Israel, donde estos últimos dos países han implementado proyectos de ley durante los últimos 10 años, promoviendo la entrevista única como el modelo predominante en investigaciones de abuso sexual infantil, y cuentan con aprendizajes y resultados dignos de considerar, además de las redes previas con profesionales conocedoras e integrantes del sistema jurídico, teniendo acceso a sus impresiones, lo cual posibilitó la consecución de los objetivos planteados.

Se utilizó como técnica de recolección de datos el análisis de información secundaria a partir de la revisión del material bibliográfico, y entrevistas semiestructuradas. Esta herramienta refiere a una pauta de preguntas tentativas que actuaron como marco de referencia, siendo así pertinente porque da espacio para temáticas emergentes no consideradas en la estructuración de la pauta y posibilita develar la importancia que le entregan a ciertas temáticas que emanan de sus discursos.

En cuanto a las entrevistas, en Suecia fue posible conversar con una experta en temáticas de bienestar infantil. El muestreo fue tipo bola de nieve, contactando a esta experta por acceso, no por selección aleatoria.

Para el caso de Israel, también se realizó una entrevista con una experta en temáticas de abuso sexual infantil, quien trabaja en la Clínica de Acompañamiento de Niños/as Víctimas de Delitos Sexuales. Lo mismo en el caso de Chile, donde se aplicaron dos entrevistas semiestructuradas a profesionales de Fundación Amparo y Justicia y, por otro lado, del Ministerio de Justicia.

Esta muestra responde al perfil de conocimiento experto por los años de experiencia en la temática 
y sus conocimientos profundos de los procesos investigativos en casos de abuso sexual infantil en sus países respectivos.

\section{Breve análisis de la información obtenida}

En primer lugar, el proyecto de ley para Chile se encuentra atravesando por la discusión base acerca de quiénes serían los profesionales idóneos para realizar la entrevista videograbada. Asimismo, a qué instituciones estas pertenecerían, siendo un punto fundamental de despejar a la hora de su aprobación:

"Originalmente el proyecto está pensado con profesionales... habian distintas posturas en esto, la personal era... yo creía que debían ser profesionales de equipos adosados a la Policía de Investigaciones, quizás departamentos al interior del CAVAS, que el CAVAS tuviera un departamento de entrevistas, separado del de pericia, separado del de reparación, que tuviera presencia nacional y que fueran profesionales que estuvieran dedicados a esto, a la entrevista videograbada, se hizo una serie de estimaciones por región, porque esto requiere que en los minutos en que ocurre una denuncia efectivamente el profesional esté disponible en la menor cantidad de tiempo posible realizando la entrevista, se requiere infraestructura, esto requiere un sistema más o menos complejo de implementar". (María Paz Rutte, Fundación Amparo y Justicia)

"Lo que pasa es que ese proyecto de ley involucra que esa toma de declaración no sea realizada por gente de la Fiscalía, o sea, que sea el fiscal... pero la persona que lleva todo, que coordine en el fondo... no sea un profesional de URAVIT como es ahora. Lo que se busca es que un organismo externo lo realice, entonces... obviamente, que esa tarea pase a otras manos... y eso significa que nuestro trabajo, que nuestra labor va a ir cambiando [...]". (Solange Garrido, Fiscalía Regional Metropolitana Sur)

Lo anterior ha sido tema de controversia entre las entrevistadas, ya que por un lado se menciona:

"[...] lo que plantean los expertos en la investigación es que, entre comillas, podría entrevistar un psicólogo, un trabajador social, un policía, o sea, lo central sería el entrenamiento, pero el entrenamiento tiene... tiene que haber un sistema de entrenamiento, no es que tú te capacites un mes y seas entrevistador, sino que tiene que haber un sistema de monitoreo de perfeccionamiento regular y continuo... y ese es el centro de esto, o sea, más que los psicólogos entrevisten mejor que los trabajadores sociales, o que lo hagan mejor los policías o que los policías no lo sepan hacer, eventualmente, cualquiera de ellos podría hacerlo, el punto... lo que pasa es que además hay que... como hay distintos protocolos, hay que tomar un protocolo que ya se está haciendo a través de un proyecto "FONDEF" de la U. de Chile con la Católica... como normalizar, estandarizar, traducir ese protocolo a la realidad chilena". (María Paz Rutte, Fundación Amparo y Justicia)

Mientras que desde la profesional del Ministerio Público se señala:

"Yo creo que lo que hay que hacer es aprovechar este recurso que hay, o sea, evidentemente viene todo un plan de fortalecimiento del Ministerio Público, porque no podemos hacer las cosas bien si tenemos poca gente... (...) complejo que se aprobara y que fuera otra institución, se removerían todos los cimientos del Ministerio Público, yo creo que mucha gente renunciaría... no le encontraría... es como empezar de nuevo podríamos decir, porque el Ministerio Público también tiene su proyecto, que lo desconozco, pero lo que se busca es que el Ministerio Público pueda usar el recurso que está acá... si no, no tiene sentido... (...) Es el fiscal el que tiene que tomar la declaración, no un ente distinto, creo yo... porque por algo tiene... porque si no al fiscal se le quitan todas las atribuciones que tiene, o sea, cada vez tiene menos... o sea, se supone que el fiscal acusa e investiga, y acá más encima ni siquiera puede investigar como corresponde...". (Solange Garrido, Fiscalía Regional Metropolitana Sur).

Aspecto en el cual desconocemos si, actualmente, se ha llegado a acuerdo, pues las discusiones más recientes realizadas en la Cámara de Senadores no han sido publicadas a la fecha de este escrito.

Según lo señalado por Dra. Evelyn Khoo, experta académica de la University of Umea en Suecia, en entrevista con fecha 16 de noviembre de 2014, "El fiscal, el psicólogo y el trabajador social están presentes al otro lado del espejo. Pueden mirar la entrevista desde el vidrio, pero no entrevistan directamente al niño", ya que quien aplica la entrevista es un policía con formación especializada. A partir del relato del NNA, los profesionales empiezan a coordinar para proveer los servicios integrales que requiere el NNA.

En el caso de Israel (cuyo entrevistado solicita mantener su identidad en reserva), son los profesionales 
como trabajadores sociales o psicólogos quienes realizan las entrevistas, pero trabajando en conjunto con la policía.

En segundo lugar, respecto a quién debiese guiar la investigación en Chile, si bien existe un acuerdo en que esta responsabilidad recaería en la figura del fiscal, los motivos y percepciones varían entre ellas. Las profesionales entrevistadas expresan:

"Ese es uno de los grandes temas, cada institución tiene su... si bien el fiscal es quien dirige la investigación y las policías son sus auxiliares... las mismas instituciones han ido incorporando... por ejemplo, la policía ha tenido una serie de capacitaciones en temas de entrevistas, la misma fiscalía tiene también un procedimiento en casos de delitos sexuales, donde el fiscal derechamente envía la orden a las policías para que no se tomen entrevistas, pero al día de hoy, a mi juicio, no hay una politica integral que dirija todas estas acciones, una politica integral de protección de la infancia, sino que lo que rigen son las políticas particulares, es decir, el interés de la fiscalía por investigar y el interés de las policías por aportar en estos medios o por tomar declaraciones". (Maria Paz Rutte, Fundación Amparo y Justicia)

"El Ministerio Público, cuando se crea esta institución es porque... tiene autonomía propia, da lo mismo el gobierno que venga... entonces yo pienso que, efectivamente, tiene que estar en manos del fiscal, porque quién mejor que él.... el que tiene que dar las directrices para la investigación y todo lo demás...". (Solange Garrido, Fiscalía Regional Metropolitana Sur)

En tercer lugar, se destacan de los modelos de Israel y Suecia aspectos positivos del tratamiento judicial para casos de abuso. Del primero, la existencia de un protocolo de entrevista basado en evidencias, que comprueban la efectividad del mismo. Mientras que del segundo, se valora el énfasis en la coordinación entre los profesionales de distintos servicios para abarcar la integralidad del fenómeno, donde los límites y las funciones claras permiten resguardar el interés superior del niño, previamente definido.

En el proceso contemplado en el proyecto de ley en Chile, no se encuentra definido con exactitud un protocolo de trabajo, a diferencia de lo que ocurre en los otros países. En Israel, éste se inicia con la designación de un profesional de los centros especializados que acompaña al NNA en todo el proceso, lo cual está especificado y no deja espacio para interpretaciones y voluntades.
En cuanto a la coordinación de profesionales en el contexto de Israel, en revisión bibliográfica y coordinación con profesional de la Clínica para Acompañar a Niños y Niñas Víctimas de Delitos (Clinic for Accompanying Child Victims of Crime), la experta Vered Windman concluye: "para reducir la victimización secundaria del NNA, las autoridades deberían implementar un sistema que asegure que los NNA ofendidos y sus familias sean informados y que reciban asistencia y explicaciones de los servicios y apoyo de forma rápida". (Windman, \& Asher, 2007, p. 321)

En la entrevista realizada a la experta de Israel, así también como en la revisión bibliográfica de la implementación del protocolo NICHD (National Institute of Child Health and Development) en casos de ASI, se comenta acerca de la creación de un centro para el acompañamiento de NNA durante el proceso investigativo, en donde un equipo de profesionales especializados aborda las dificultades que puedan presentar, representando sus intereses y realizando terapia reparatoria.

Tanto Israel como Suecia han implementado estos centros especializados para NNA en situaciones de ASI, para acompañarlos durante el proceso investigativo. En cuanto al procedimiento, se encontró una división de las opiniones de los profesionales frente a la propuesta de una entrevista única forense; si bien todos los profesionales manifestaron su interés en el niño y en no exponerlo a una victimización secundaria, algunos expertos comentan que algunos, sobre todo en casos de abuso sexual intrafamiliar, requieren construir un vínculo más profundo antes de develar, razón por lo cual sugiere entrevistas repetitivas. Si bien el protocolo NICHD y los profesionales entrevistados en Israel y Suecia no definieron un plazo para realizar estas entrevistas, ambas manifestaron que "lo más rápido posible" después de la denuncia.

Como cuarto, y último punto, se expresa, sin ansias de ser reiterativo, la necesidad de una Ley de Protección Integral de la Infancia, que no sea expuesta como asistencialismo en pro de sujetos vulnerables, sino que sea consciente, conocedor y respetuoso de los derechos de los mismos.

De este modo, el país podría responder a la deuda histórica, tal como lo han hecho Suecia e Israel.

\section{Conclusiones}

El abuso sexual contra NNA es considerado a nivel mundial un delito con características particulares, por lo se requiere que los sistemas penales con- 
tengan estructuras, procedimientos y mecanismos adecuados para facilitar la exteriorización de denuncias y optimizar las oportunidades existentes para la recolección de las pruebas disponibles que apoyen el procedimiento penal.

La revictimización se genera por problemas estructurales de articulación del sistema, pero estos se encuentran representados en personas que con sus conductas altamente vulneradoras y sus creencias estigmatizadoras no contribuyen a los procesos de denuncia y mucho menos a los reparatorios. Esto porque exigen de parte de una niña o un niño un relato que entregue detalles precisos y periféricos de una experiencia traumática, sin considerar la afectación emocional o los recursos cognitivos de la etapa del desarrollo de éste, desplegando acciones descontextualizadas.

Según los antecedentes recabados de las entrevistas con profesionales que trabajan en temáticas de ASI, la sociedad civil estaría a favor de que los profesionales a cargo de aplicar entrevistas videograbadas provengan de un servicio externo especializado en delitos sexuales contra NNA. Mientras que desde la percepción obtenida del servicio público, este proceso debiese continuar en manos de la Fiscalía.

Ahora bien, con base en la información internacional recabada en el marco de esta investigación, es posible sugerir la creación de centros integrales para casos de ASI, que mediante turnos de trabajo brinden un servicio en todo momento; contemplando toma de denuncias que no impliquen interrogatorio directo, tal como se expone en el proyecto de ley. Esencialmente, Suecia e Israel combinan los mundos privados y públicos, involucrando a los actores estatales con organismos privados, existiendo un mayor nivel de coordinación y sincronía entre todos los involucrados. Es preciso mencionar que en este punto se requeriría de la sensibilización de los profesionales que rodean los procesos de investigación para que el objetivo de evitar la revictimización sea alcanzado.

Se propone así, que tal como sucede en Suecia, todos quienes se encuentren trabajando en este tipo de casos se ajusten a funciones previamente establecidas, distintas y complementarias, que permitan articular el engranaje judicial en pro de evitar la vulneración que produce el sistema actual, como también es relevante consensuar una definición de qué se entenderá por el interés superior del niño, explicitando los actores y funciones principales que procurarán su resguardo.

Desde la experiencia internacional de la entrevista videograbada, considerando el contexto chileno y el proyecto de ley, es que se pueden sugerir los siguientes elementos:

\section{a. Políticas flexibles:}

Según lo identificado por los profesionales, a nivel nacional como internacional, es necesario incorporar políticas y prácticas flexibles en lo penal, ya que, en algunos casos, la entrevista única podría llegar a una develación formal y colaborativa con el proceso. No obstante, hay quienes, por su edad o sus características personales, requieren establecer más rapport con el profesional, requiriendo múltiples entrevistas para relatar la experiencia del abuso sexual.

Por tanto, con el fin de proveer a cada NNA un espacio y oportunidad de ser escuchado en su propio relato, es que sería pertinente la posibilidad de realizar una entrevista única, la cual pudiera ser extendida o repetida según las necesidades de cada caso.

Por ejemplo, el proyecto de ley actual sugiere un plazo de 72 horas después de la denuncia para realizar la entrevista videograbada con el NNA, lo cual debiese tener la salvedad de considerar las necesidades del NNA, facilitando el proceso investigativo desde la flexibilidad de los entrevistadores, ya que podrían existir quienes emocionalmente requieran más tiempo, debiendo considerar ello y brindar la posibilidad de ser escuchados acorde a sus procesos personales más que a un parámetro estandarizado.

Asimismo, puede ser que dos entrevistas no sean suficientes, conociendo el comportamiento de los NNA y la forma en que se dan estos procesos. Se sugiere abrir la posibilidad de realizar más entrevistas según sea el caso.

\section{b. Centros especializados de ASI:}

Desde las experiencias internacionales, lo ideal sería tener centros especializados en cada región, contando con equipos profesionales de trabajadores sociales y psicólogos, capacitando también a los carabineros locales, para facilitar el proceso investigativo y de acompañamiento a NNA.

Como el interés superior del niño deja un alto margen para la interpretación, así como también el concepto de criterio profesional, cada servicio desde sus protocolos procede con sus acciones, y el único punto en común donde se cruzan es en el relato esperado; se hace preciso puntualizar en la necesidad de unificar los protocolos de las distintas entidades, cohesionándolos en uno. 
Al ser la denuncia un paso complejo y desestructurante para las familias y para el NNA afectado, rodeado de miedos y falsas creencias, la demora en los procesos sólo agrava las condiciones y aumenta la ansiedad al dar espacio para ejercer presiones desde las distintas esferas, aumentando la probabilidad de retractación. Por tanto, considerando la evidencia empírica desde la revisión bibliográfica internacional, así como también la coordinación con profesionales de Suecia e Israel, se podría hipotetizar que al implementar estos centros en Chile pudieran bajar los niveles de ansiedad de la familia y el NNA previniendo la retractación.

\section{c. Profesionales con formación y experiencia en ASI:}

La implementación del proyecto de ley significará un cambio de las políticas y prácticas actuales, implicando una transformación en la mirada de los profesionales a cargo de realizar las entrevistas investigativas en situaciones de abuso. En virtud de esto, considerando lo analizado desde el protocolo NICHD de Israel y la evidencia empírica, es que se estima pertinente sugerir cursos de especialización y formación para profesionales, antes de empezar a implementar el nuevo modelo de entrevista videograbada.

Asimismo, la formación/especialización de los profesionales que realizan las entrevistas es necesaria para minimizar los efectos de la victimización secundaria, abordar las dificultades y el impacto del daño asociado a las vulneraciones en la esfera de la sexualidad. La clave es que el profesional que sea (policía, trabajador social, psicólogo o fiscal) tenga formación y especialización en protocolo para entrevista única, así también reconociendo aspectos del fenómeno de ASI en el contexto chileno.

\section{d. Protocolo estandarizado y universal:}

Conjuntamente, los proyectos en los tres países analizados refieren a la importancia de establecer un protocolo estandarizado para la entrevista videograbada, sugiriendo aquellos basados en evidencia (Vandervort, 2006; Orback et al., 2000; Cross et al., 2007; Campbell et al., 2001; Orbach et al., 2000). No obstante, si bien el proyecto de ley chilena refiere a éste, no especifica cómo sería, dejando espacio para la interpretación e implementación subjetiva, según los entrevistadores.

Desde la experiencia internacional, el protocolo NICHD ha resultado efectivo, así también como la entrevista cognitiva y la entrevista "Step-wise"
(Lindberg et al., 2010). Por tanto, sería beneficioso realizar investigaciones para conocer el contexto chileno, con el fin de evaluar la efectividad de estas entrevistas a fin de unificar criterios.

\section{e. Hacia una política integral de infancia:}

En conjunto con lo anterior, hay que examinar el marco sociojurídico de la infancia en los distintos países, con el fin de implementar políticas específicas en la temática. Al evaluar el proyecto de ley, es esencial también considerar la imagen de la infancia actualmente en Chile, así también como la definición legal de la niñez.

La revictimización y victimización secundaria reflejan la estigmatización, la etiqueta y la imagen de los niños dentro de la sociedad chilena. A pesar de haber ratificado CDN, todavía no hemos implementado una política integral de infancia, permaneciendo los NNA bajo la misma mirada legal de antes, no logrando hasta la fecha generar políticas más integrales y empoderantes.

Desde el contexto sueco, aprobaron el Acta de Servicios Sociales de 1980 (Swedish Social Services Act), enfocando los servicios sociales hacia programas preventivos y la infancia, socializando estos servicios hacia todos los sectores de la sociedad, independiente de los propios recursos familiares. En 1993, crearon el Ombudsman del Niño, una entidad estatal, con el fin de resguardar los derechos de los NNA, así también como representar y acompañarlos en procesos legales (Andersson, 2002). Mientras cada municipalidad tiene cierta autonomía para poder elegir e implementar programas sociales, el gobierno estatal, a través del Ombudsman del Niño, regula y monitorea el cumplimiento de las leyes y regulaciones federales.

Con respecto a Israel, el Servicio de Niños y Jóvenes (The Service for Children and Youth), administrado por el Ministerio de Servicios Sociales, fue creado después de la CDN, con el fin de resguardar los derechos, así también para monitorear las agencias que intervienen directamente con NNA (Alfandri, 2010; Windham \& Asher, 2007). Es importante mencionar que en los últimos años, el gobierno israelí ha recibido mucha crítica desde el mundo internacional, respecto a su falta de resguardo a los derechos de los niños palestinos. No obstante, desde la estructura y la implementación del Servicio de Niños y Jóvenes, se puede identificar elementos positivos que pudieran ser extrapolados hacia otros contextos, ya que desde el marco jurídico, los niños en Israel también son sujetos de derechos, con polí- 
ticas comprensivas para resguardar estos.

Por lo tanto, es necesario reflexionar sobre la estructura más macro de la sociedad chilena, ya que para poder generar un cambio real, surge la necesidad de cambiar la imagen de la niñez.

El desafío, además del Estado, queda en quienes participamos en los procesos interventivos y en las distintas áreas de trabajo con infancia, en donde se debe abordar la vulneración de derecho constitutivo de delito como es el abuso sexual, desde la sensibilización de los diferentes actores de la comunidad, la prevención y la acción en relación al establecimiento de protocolos; en este sentido, es preciso educar y visibilizar.

\section{Bibliografía}

ALFANDARI, R. (2010), Decision Making in Child Protection: The Impact of an Analytical Framework. Desde: http:// www.academia.edu/691227/Decision_Making_in_Child_ Protection_The_Impact_of_an_Analytical_Framework. Extraído el 10 de diciembre de 2014.

ALZATE, M. (2002). El "descubrimiento" de la infancia (I): historia de un sentimiento". Revista Ciencias Humanas, $N^{\circ}$ 30. Desde: http://www.utp.edu.co/ chumanas/ revistas/revistas/rev30/alzate. Extraído el 25 de noviembre de 2014

AMNESTY INTERNATIONAL (2006), Case Closed: Rape and Human Rights in the Nordic Countries. Desde: http://www.amnesty.dk/sites/default/files/mediafiles/44/ case-closed.pdf. Extraído el 18 de diciembre de 2014.

ANDERSSON, G. (2002), Partnerships for Children and Families Project: Child and Family Welfare in Sweden. Desde: https://www.wlu.ca/documents/7208/Andersson. pdf. Extraído el 18 de noviembre de 2014.

ASSOCIATION OF RAPE CRISIS CENTER IN ISRAEL (2014). 2007 Data and Statistics. Desde: http://www. 1202.org.il/English/template/default. asp?siteId=6. Extraído el 8 de diciembre de 2014

BALA, N. (1990), Double Victims: Child Sexual Abuse and the Canadian Criminal Justice System. Queens Law Journal Vol. 3.

BRUCK, M., CECI, S., \& SHUMAN, D. (2007) Disclosure of Child Sexual Abuse: A Review of the Contemporary Empirical Literature. In Child Sexual Abuse: Disclosure, Delay and Denial. Psychology Press.

CAMPAÑA NO ME PREGUNTEN MÁS (2014). Entrevista Videograbada. Desde: http:nomepreguntenmás. cl/docs/entrevistasvideograbadas.pdf. Extraído el 1 de diciembre de 2014.

CAMPBELl, R., WASCO, S., AHRENS, C., SEFL, T., \& BARNES, H. (2001), Preventing the "Second Rape": Rape Survivors' Experiences with Community Service
Providers. Journal of Interpersonal Violence, Vol. 16 (12) Pp. 1239-1259.

CECI, S. J \& BRUCK, M. (1995), Jeopardy in the courtroom: A scientific analysis of children's memory. Washington, D.C. American Psychological Association.

COMISIÓN INTERECLESIAL DE JUSTICIA Y PAZ (2010). Los efectos psicosociales de la revictimización. Desde: http://justiciaypazcolombia.com/Los-efectospsicosociales-de-la. Extraído el 10 de diciembre de 2014

CROSS, T., JONES, L., WALSH, W., SIMONE, M., \& KOLKO, D. (2007), Child forensic interviewing in Children's Advocacy Centers: Empirical data on a practice model. Child Abuse and Neglect, Vol. 31, Pp. 1031-1052.

GOETZ, J. \& LECOMPTE, M. (1988). Etnografía y diseño cualitativo en investigación educativa. Madrid: Editorial Morata

HERNÁNDEZ, M. (2010). Niños y niñas que han vivido maltrato grave y/o abuso sexual del servicio psicológico de koinomadelfia. El Observador: Revista especializada en temas de infancia y adolescencia del Servicio Nacional de Menores. $\mathrm{N}^{\circ}$ 6, pp. 48 - 61.

HERSHKOWITZ, I. \& TERNER, A. (2006), The Effects of Repeated Interviewing on Children's Forensic Statements of Abuse. Applied Cognitive Psychology, Vol 21. Pp. 1131-1143.

ISRAELI PENAL CODE (1977), Desde: http://www. omct.org/files/2004/07/2409/esp_2001_05_israel.pdf. Extraído el 10 de diciembre de 2014.

JAUDES, P \& MATRONE, M. (1992), Interdisciplinary evaluations of alleged sexual abuse cases. Pediatrics, Vol. 89 (6) Pp. 1164-1168.

JONES, D. (1991), Professional and clinical challenges to the protection of children. Child Abuse \& Neglect. Vol 15 (1) Pp. 57-66

IBACETA, F. (2007). Agresión Sexual en la Infancia y Viaje al Futuro: Clínica y Psicoterapia en la Edad Adulta. Terapia Psicológica, Vol. 25, N² 2, 189-198.

LINDBERG, M., TANTALO CHAPMAN, M., SAMSOCK, D., THOMAS, S., LINDBERG, A. (2010), Comparisons of Three Different Investigative Interview Techniques with Young Children. The Journal of Genetic Psychology: Research and Theory on Human Development. Vol. 164 (1) Pp. 5-28.

LAW LIBRARY OF CONGRESS (2014), Children's Rights: Israel. Desde: http://www.loc.gov/law/help/ child-rights/israel.php. Extraído el 14 de diciembre de 2014

MAFFIOLETTI, F. Y HUERTA, S. (2011). Aproximación fenomenológica de los delitos sexuales en Chile: La realidad nacional. Revista Jurídica del Ministerio Público, No 47. Desde: http://www.fiscaliadechile.cl/Fiscalia/ar 
chivo?id=2016\&pid=124\&tid=1\&d. Extraído el 6 de diciembre de 2014.

MINISTERIO DE JUSTICIA (2000). Código Procesal Penal de Chile.

MINISTERIO PÚBLICO (2012). GEV: Guía de Entrevista Investigativa con Niños, Niñas y Adolescentes Víctimas de Delitos Sexuales. Unidad Especializada en delitos sexuales y violentos. Fiscalía Nacional del Ministerio Público de Chile.

MINISTRY OF JUSTICE (1999). Swedish Penal Code. Government Office of Sweden.

MOSSIGE, S., AINSAAR, M. SVEDIN, C. (2007). The Baltic Sea regional Study on Adolescents' Sexuality. Norsk institutt for forskning om oppvekst, velferd og aldring NOVA Rapport 18/2007.

ORBACH, Y., HERSHKOWITZ, I., LAMB, M. \& STERNBERG, K. (2000), Assessing the value of structured protocols for forensic interviews of alleged child abuse victims. Child Abuse \& Neglect, Vol. 24 (6), Pp. 733 752.

PIPE, M., LAMB, M., ORBACH, Y., \& CEDERBORG, A. (2007), Child Sexual Abuse: Disclosure, Delay and Denial. Psychology Press.

PRIEBE, G. \& SVEDIN C.G. (2009). Prevalence, characteristics and associations of sexual abuse with sociodemographics and consensual sex in a population-based sample of Swedish adolescents. Journal of Child Sexual Abuse, 18 (1), 19-39. DOI: 10.1080/10538710802584635

RAPE CRISIS CENTERS IN ISRAEL (2007). Report of 2007 Data and Statistics. Desde: http://www.1202.org. il/English/template/default.asp?siteId $=1$ \&maincat $=18$. Extraído el 18 de diciembre de 2014.

SAN MARTÍN, M. (2014). Medidas de protección para niños, niñas y adolescentes víctimas o testigos. Una cuestión de principios. Revista jurídica del Ministerio Público. N 59, pp. 187 - 204.

SAVE DE CHILDREN (s/f). Abuso Sexual Infantil: Manual de formación para profesionales Save the Children. Desde: http://www.margen.org/docs/curso27-8/ Unidad1/1-Que\%20es\%20Abuso\%20Sexual\%20Infantil.doc. Extraído el 30 de noviembre de 2014.

SENADO (2014a). Ingreso del Proyecto de Ley: Regulación de entrevistas grabadas en video $y$, otras medidas de resguardo a menores de edad víctimas de delitos sexuales. Primer trámite constitucional. Desde: http://www.camara.cl/pley/pley_detalle. aspx?prmID=9657\&prmBL=9245-07. Extraído el 30 de octubre del 2014.

SENADO (2014b). Discusión General de Proyecto de Ley: Regulación de entrevistas grabadas en video y, otras medidas de resguardo a menores de edad víctimas de delitos sexuales. Desde: http://www.camara.cl/pley/ pley_detalle.aspx?prmID=9657 \&prmBL=9245-07. Extraído el 30 de octubre de 2014
STAKE, R. (1998). Investigación con estudio de casos. Madrid: Editorial Morata.

THEMELI, O. \& PANAGIOTAKI, M. (2014). Forensic Interviews with Child Victims of Sexual Abuse: The Role of the Counselling Psychologist. The European Journal of Counselling Psychology. Vol. 3 (1).

UNDERWAGER, R. \& WAKEFIELD, H. (1990), How to Avoid Secondary Victimization in Child Sexual Abuse Investigations. Presented at the Annual Conference of the National Council for Children's Rights. Arlington, VA. Desde: http://files.eric.ed.gov/fulltext/ED330445. pdf. Extraído el 14 de diciembre de 2014.

UNICEF (2005), United Nations Office on Drugs and Crime, Justice in Matters involving Child Victims and Witnesses of Crime. New York. Desde: http://www. unicef.org/albania/Justice_in_matters.pdf Extraído el 14 de diciembre de 2014

UNICEF, UDP (2006), Niños, niñas y adolescentes víctimas de delitos sexuales, en el marco de la reforma procesal penal. Informe Final. Fondo de las Naciones Unidad para la Infancia (Of. para Chile y el Conosur) / Instituto de Investigación en Ciencias Sociales - Universidad Diego Portales. Desde: http://www.unicef.cl/ archivos_documento/173/Informe\%20final.pdf. Extraído el 30 de noviembre de 2014.

VANDERVORT, F. (2006), Videotaping Investigative Interviews of Children in Cases of Child Sexual Abuse: One Community's Approach. The Journal of Criminal Law and Criminology, Vol. 96 (4), Pp. 1353-1416.

WINDAM, V. \& ASHER, B. (2007), Secondary Victimization of Children in Israel and the Child's Perspective. International Review of Victimology, Vol. 14 (3) Pp. 321 336. 


\section{ANEXOS}

\section{ANEXO 1: TABLA COMPARATIVA DEL PROCESO INVESTIGATIVO EN CADA PAÍS}

\begin{tabular}{|c|c|c|c|}
\hline País & $\begin{array}{l}\text { ¿Quién realiza } \\
\text { investigación? }\end{array}$ & $\begin{array}{l}\text { ¿Quién realiza } \\
\text { la entrevista? }\end{array}$ & ¿Cómo es el proceso? \\
\hline Chile & $\begin{array}{l}\text { Fiscal está a cargo de la } \\
\text { investigación. }\end{array}$ & $\begin{array}{l}\text { En el proyecto de ley aún no } \\
\text { está definido, es parte de las } \\
\text { discusiones que se están lle- } \\
\text { vando a cabo. }\end{array}$ & $\begin{array}{l}\text { Una vez realizada la denuncia, se disponen de } 72 \\
\text { horas para realizar la primera entrevista (entre- } \\
\text { vista investigativa) por el profesional "idóneo", la } \\
\text { cual será dirigida por el fiscal, quien puede inter- } \\
\text { venir pero sólo a través del entrevistador, y quien } \\
\text { podría suspender esta entrevista si así lo estimare } \\
\text { conveniente, proponiendo fecha para una nueva } \\
\text { oportunidad. } \\
\text { Posteriormente, se realiza una segunda entrevista } \\
\text { (por el mismo entrevistador), la entrevista judicial } \\
\text { la cual servirá de prueba para el juicio que se lleve } \\
\text { a cabo, la cual será dirigida por el juez de garantía. }\end{array}$ \\
\hline Suecia & $\begin{array}{l}\text { Fiscal está a cargo de la } \\
\text { investigación. }\end{array}$ & $\begin{array}{l}\text { Policía especializada realiza } \\
\text { entrevista en centros espe- } \\
\text { cíficamente habilitados para } \\
\text { entrevistas forenses. }\end{array}$ & $\begin{array}{l}\text { Una entrevista forense (grabada, para que el fisca } \\
\text { y equipo profesional pueda revisarla y construir su } \\
\text { caso/plan de intervención acorde). } \\
\text { En el juicio, un juez titular y tres jueces elegidos por } \\
\text { la comunidad deciden el veredicto. Incorporan la } \\
\text { grabación, para evitar la re-victimización del niño. }\end{array}$ \\
\hline Israel & $\begin{array}{l}\text { Fiscal, en coordinación con } \\
\text { los profesionales tratantes, } \\
\text { asignados para acompañar- } \\
\text { le al niño/niña/adolescente } \\
\text { durante el proceso investi- } \\
\text { gativo. }\end{array}$ & $\begin{array}{l}\text { Trabajadores sociales o psi- } \\
\text { cólogos con formación y } \\
\text { experiencia en materia de } \\
\text { abuso sexual infantil. Ellos } \\
\text { hacen sugerencias al fiscal. }\end{array}$ & $\begin{array}{l}\text { Utilizan el protocolo NICHD, en donde priorizan } \\
\text { una entrevista única; no obstante, existe la posibi- } \\
\text { lidad de repetir entrevistas, siempre que el niño no } \\
\text { logra colaborar con el proceso la primera vez. }\end{array}$ \\
\hline
\end{tabular}

Fuente: Elaboración propia. 


\section{ANEXO 2: PAUTA DE ENTREVISTA NACIONAL}

Nombre:

País de residencia:

Profesión:

Ocupación actual:

Institución de pertenencia:

Años que lleva trabajando en temática de infancia/derechos del NNA:

Estimado/a, mi nombre es XX, me encuentro cursando el Diplomado de Intervención en Abuso Sexual Infantil de la Pontificia Universidad Católica de Chile, en el cual estamos realizando un estudio denominado "Avanzando hacia la protección integral de los derechos de la infancia: Una mirada al proyecto de ley que regula entrevistas grabadas en video y otras medidas de resguardo a menores de edad víctimas de delitos sexuales". El propósito de la siguiente conversación es conocer su opinión con relación a la técnica de Entrevista Única para la obtención del relato de NNA víctimas de delitos sexuales en Chile, a partir de su conocimiento y experiencia profesional.

Ésta será grabada a fin de respaldar la información, y solo se utilizará para fines investigativos, cualquier otro uso le será comunicado previamente.

Agradecemos de antemano su colaboración.

1. Describa brevemente la labor que usted, y la institución a la cual pertenece, realizan en relación a la protección de los derechos de los NNA.

2. Podría comentarnos desde su conocimiento ¿cuál es el procedimiento en Chile para casos de denuncias por abuso sexual infantil?

3. ¿Cuál es el organismo que se hace cargo del proceso investigativo? Sondear aplicabilidad a partir de respuesta anterior

4. De acuerdo a su percepción, ¿cuál debería ser el rol del Estado, a través de sus distintas figuras institucionales, en la implementación de la entrevista única?

5. Podría contarnos qué sabe usted acerca del proceso de aplicación de entrevistas a niños y niñas que han sufrido alguna vulneración de sus derechos en la esfera de la sexualidad. ¿Y cuál es su opinión respecto a ello?

6. Respecto a la entrevista única, ¿qué tipo de formación/especialización cree usted que se requieren para realizar ésta?

7. Según su opinión, ¿qué falencias o dificultades se pueden presentar en Chile, a la hora de implementar esta técnica?

8. ¿Qué sugerencias o cambios haría en líneas generales, al proyecto de ley que regula entrevistas grabadas en vídeo y otras medidas de resguardo a menores de edad víctimas de delitos sexuales? 


\section{ANEXO 3: PAUTA DE ENTREVISTA INTERNACIONAL}

Nombre:

País de residencia:

Profesión:

Ocupación actual:

Institución de pertenencia:

Años que lleva trabajando en temática de infancia/derechos del NNA:

Estimado/a, mi nombre es XX, me encuentro cursando el Diplomado de Intervención en Abuso Sexual Infantil de la Pontificia Universidad Católica de Chile, en el cual estamos realizando un estudio denominado "Avanzando hacia la protección integral de los derechos de la infancia: Una mirada al proyecto de ley que regula entrevistas grabadas en video y otras medidas de resguardo a menores de edad víctimas de delitos sexuales". El propósito de la siguiente conversación es conocer su opinión en relación a la implementación de la técnica de Entrevista Única para la obtención del relato de NNA víctimas de delitos sexuales, a partir de su conocimiento y experiencia profesional.

Ésta será grabada a fin de respaldar la información, sólo se utilizará para fines investigativos, cualquier otro uso le será comunicado previamente.

Agradecemos de antemano su colaboración.

1. Describa brevemente la labor que usted, y la institución a la cual pertenece, realizan en relación a la protección de los derechos de los NNA.

2. ¿Cuál es el procedimiento en su país/Estado en casos de denuncias por abuso sexual infantil?

3. ¿Cuál es el organismo que se hace cargo del proceso investigativo?

4. ¿Cómo responde el Estado frente a denuncias de abuso sexual infantil? ¿Cuál es su rol?

5. ¿Cuál es el marco legal en que surge la entrevista única en su país/Estado como técnica para abordar estos casos?

6. ¿Quién son los profesionales encargados de realizar esta entrevista? ¿Tienen una formación particular?

7. Según su opinión, ¿cuáles fueron las principales dificultades para implementar esta técnica?

8. ¿Cómo describiría la experiencia de la implementación de entrevista única en su país/Estado? (Comentarios sobre los beneficios y perjuicios desde la opinión personal)

9. ¿Qué sugerencias o cambios haría a esta técnica? 
